\title{
BISTUA
}

\section{Expresión propuesta para el diseño de voladuras en túneles de carretera, caso colombiano}

\author{
Proposed expression for the road tunnels blasting design, Colombian case.
}

\author{
Álvaro Correa Arroyave ${ }^{a}$; Jorge Eric Rueda Fonseca ${ }^{b}$, Jeison Fabián Rodríguez Beltrán ${ }^{c}$ \\ ${ }^{a}$ Ingeniero de Minas y Metalurgia, Doctor-Ingeniero en Mecánica de Rocas, Especialista en Técnicas de voladura a cielo abierto y subterráneo, \\ Docente Universidad Santo Tomás, Bogotá; Docente Escuela de Ingenieros Militares, Bogotá, Colombia, \\ ${ }^{b}$ Geólogo, Especialista en Técnicas de voladura en obras de ingeniería civil y militar, Escuela de Ingenieros Militares, Bogotá, Colombia \\ ${ }^{c}$ Ingeniero Civil, Universidad Santo Tomás, Bogotá, Colombia, jfabianrb@gmail.com
}

Correspondencia: alvarocorreaa@usantotomas.edu.co ; jorge.eric.rueda.fonseca@gmail.com

Recibido: Enero 16, 2021. Aceptado: Febrero 10, 2021. Publicado: Junio 19, 2021

\section{Resumen}

El diseño de una voladura tanto a cielo abierto como subterráneo es un proceso que conlleva observación, análisis y control de todas las variables que en él intervienen. Es condición mínima el conocimiento de la roca que se va a volar, así como las características de fragmentación del macizo, y, por supuesto el tipo de explosivo y las características geométricas de la voladura. Posteriormente, en función de la experiencia adquirida en el sitio de la voladura, se recurre a algunas de las expresiones propuestas por los investigadores en el campo de la perforación y voladura y se procede finalmente al análisis de sus resultados, los cuales deberían estar acordes con las expectativas generadas. En Colombia, por diversas razones no se siguen estos protocolos y se procede a realizar diseños sin el mínimo conocimiento ni de la roca intacta (más allá de su nombre geológico, el que a veces tampoco se corresponde) ni de las características estructurales del macizo rocoso. El presente artículo ilustra un procedimiento fundamentado en el seguimiento riguroso a los parámetros de una buena voladura, basado en la utilización de una expresión propuesta la cual pretende tomar en cuenta las variables más importantes para el diseño de una voladura eficiente, teniendo en cuenta las características físico-mecánicas de las rocas colombianas. Pretendemos que la expresión propuesta sea tenida en cuenta, así como sus resultados, los cuales permitirán ajustar las constantes que hacen parte de ella. Los autores de la presente investigación nos encontramos dispuestos a recibir los comentarios, así como los resultados a fin de analizarlos y proceder a las mejoras a que haya lugar.

Palabras clave: RMR, Burden, RQD, RMRb, Resistencias dinámicas de las rocas, Voladura subterránea, Características físico-mecánicas de rocas colombianas.

\section{Introducción}

La determinación de los parámetros de una voladura, y particularmente su burden, espaciamiento y retaque, parecen responder a expresiones que funcionan bien tanto en trabajos a cielo abierto como subterráneos, según se desprende de la bibliografía técnica disponible; Carlos López Jimeno [1] hace un interesante resumen al citar 21 de las expresiones más

\section{Abstract}

The design of a blasting, both open pit and underground, is a process that involves observation, analysis, and control of all the variables involved. A minimum condition is the knowledge of the rock to be blasted, as well as the fragmentation characteristics of the massif, and, of course, the type of explosive and the geometric characteristics of the blasting. Subsequently, based on the experience acquired at the blasting site, some of the expressions proposed by researchers in the field of drilling and blasting are used and finally the results are analyzed, which should be in accordance with the expectations generated. In Colombia, for various reasons these protocols are not followed, and designs are made without the minimum knowledge of either the intact rock (beyond its geological name, which sometimes does not correspond either) or the structural characteristics of the rock mass. This article illustrates a procedure based on the rigorous monitoring of the parameters of a good blasting, based on the use of a proposed expression which aims to consider the most important variables for the design of an efficient blasting, considering the physical and mechanical characteristics of Colombian rocks. We intend that the proposed expression be considered, as well as its results, which will allow adjusting the constants that are part of it. The authors of this research are willing to receive comments and results to analyze them and proceed to the necessary improvements.

Keywords: Burden, RQD, RMRb, Dynamic rock strengths, Underground blasting, Physical-mechanical characteristics of Colombian rocks.

frecuentemente utilizadas. Un breve y rápido análisis de éstas permite concluir que algunas de ellas solo hacen intervenir parámetros geométricos, otras, solo la densidad del explosivo y de la roca, otras más, velocidades del sonido en explosivo y roca, y algunas más elaboradas, parámetros de fragmentación del macizo rocoso manifiestos en el RQD. Mas, lastimosamente, cada vez que estas expresiones son 
más elaboradas, menos utilización parecen tener, lo cual, a nuestro sentir, se debe a la falta de investigación de las propiedades de las rocas y de los macizos rocosos que ingresan en ellas, lo cual da lugar a diseños ineficientes, costosos y, muy posiblemente, ambientalmente cuestionables.

Por otro lado, en ninguna de las expresiones consultadas, se hacen intervenir ni el tamaño de la sección, ni el efecto del estado de confinamiento del macizo rocoso en el que se encuentra inmerso el túnel, y si bien para resolver el problema de las secciones muchas de las expresiones vienen complementadas con tablas en las que se tiene en cuenta ese aspecto, al menos en nuestra investigación, no conseguimos ninguna expresión que hiciera alusión al efecto de los esfuerzos de campo y su influencia en el diseño del patrón de perforación y voladura subterránea.

Realizando un ejercicio teórico en el que nos dimos a la tarea de hallar el burden, y a partir de él, los demás parámetros de diseño de una voladura subterránea, utilizando varias de esas expresiones, haciendo intervenir en cada caso un valor real para el parámetro solicitado, encontramos, por ejemplo, para el burden, valores entre 1,2 y 9,0 m, con una tendencia a incrementar el valor del burden en la medida que se realiza una mejor caracterización de la roca intacta y del macizo rocoso.

Probamos igualmente con algunas expresiones citadas en la bibliografía técnica en donde encontramos por ejemplo una expresión citada por Peña Castillo, Manuel F. [2] expresión que se transcribe:

$$
\begin{gathered}
B=0.684 D \cdot F ; \text { con } F=5028 /\left(I_{e} V_{d} / I_{r}\right)^{0.33} \\
\text { donde, } \quad I_{e}=\rho_{e} V_{d} \quad \text { y } \quad I_{r}=\rho_{r} V_{d}
\end{gathered}
$$

Siendo:

Ir: $\quad$ Impedancia de la roca

$\mathrm{I}_{\mathrm{e}}$ : Impedancia del explosivo

$\mathrm{V}_{\mathrm{d}}$ : Velocidad del sonido en la roca y en el explosivo

D: Diámetro de perforación

Finalmente, también hicimos el ejercicio con la expresión propuesta por Ojeda Mestas [3], la cual hace intervenir el RQD, así como un factor de seguridad dependiendo de sí el burden es para voladura de arranque $(\mathrm{Fs}=6)$, voladura de ayuda $(\mathrm{Fs}=5)$ o voladura de producción $(\mathrm{Fs}=2)$.

Siendo:

$$
B=D \cdot\left(\frac{P_{o} D}{F_{s} \cdot \sigma_{r} \cdot R Q D}+1\right)
$$

B: Burden

D: Diámetro del taladro

PoD Presión de detonación del explosivo

Fs: $\quad$ Factor de seguridad

$\sigma_{r}: \quad$ Resistencia a la compresión de la roca

RQD: Índice de calidad de la roca

\section{Ingeniería de voladura}

De las expresiones analizadas se desprende, como era de esperar, que un buen diseño debería incluir tanto propiedades de la roca (o mejor, del macizo rocoso, las cuales son más difíciles de determinar), como del explosivo y, por supuesto de ciertos parámetros geométricos como diámetro del barreno, confinamiento de la carga y llenado del barreno.

En este sentido entonces, bien vale la pena hacer una corta disertación de estos parámetros:

\subsection{Propiedades estáticas de la roca intacta}

El proceso de perforación y voladura está compuesto por dos operaciones ingenieriles que demandan diseños, cálculos, seguimiento y retroalimentaciones continuas, lo cual conlleva a considerarlas como haciendo parte de una ingeniería de perforación y por ende, una ingeniería de voladura, en donde la supervisión de las operaciones se convierte en el alma del proceso, avisando con anterioridad los ajustes que pudieran hacer falta para tener en cuenta otros parámetros no incluidos en los cálculos diarios tales como las condiciones de esfuerzos in-situ o la presencia de pliegues y fallas activas las cuales influirán en los resultados.

La bibliografía técnica, lo advierte los parámetros de la roca y del macizo rocoso que intervienen en una buena perforación y voladura son, para la perforación: dureza, resistencias, elasticidad, plasticidad, abrasividad, textura y estructura, y para la voladura: densidades, resistencias dinámicas, porosidad, fricción interna, conductividad, composición y explosiones secundarias de polvo. Por su parte, las propiedades de los macizos rocosos que mayor incidencia tienen son: litología, fisuras preexistentes, presencia de agua, esfuerzos in-situ y temperatura.

Las caracterizaciones más frecuentemente realizadas en rocas son las determinaciones de las resistencias a compresión simple y tracción indirecta, las densidades y las velocidades sónicas, con las cuales ya se pueden hacer ciertos tratamientos de datos para intuir las características de los macizos rocosos.

En ensayos realizados en rocas colombianas, en particular en una cuarcita wollastonítica y un gneis wollastonítico del macizo metamórfico de Norcasia, encontramos los siguientes comportamientos medios:

Cuarcita wollastonítica, $\mathrm{MPa}: \mathrm{RC} / \mathrm{Rt}=179,46 / 14,96=12$

Velocidad sónica: $5.400 \mathrm{~m} / \mathrm{s}$

Densidad: $2,84 \mathrm{~g} / \mathrm{cm}^{3}$

Gneis wollastonítico, MPa: RC/Rt = 224,29/16,66 = 13,50

Velocidad sónica: $5.888 \mathrm{~m} / \mathrm{s}$

Densidad: $3,73 \mathrm{~g} / \mathrm{cm}^{3}$ 


\subsection{Resistencias dinámicas de la roca intacta}

Transcribimos de la Ref. [4] lo siguiente: "Las resistencias estáticas a compresión y a tracción se utilizaron en un principio como parámetros indicativos de la aptitud de la roca a la voladura. Así se definió el Índice de Volabilidad (Hino, 1959) como la relación Rc/Rt de modo que, a un mayor valor, resultaría más fácil fragmentar el material. El tratamiento racional de los problemas reales obliga a considerar las resistencias dinámicas, ya que éstas aumentan con el índice de carga (Rinehart, 1958, Persson et al., 1970) pudiendo llegar a alcanzar valores entre 5 y 13 veces superiores a las estáticas. Cuando la intensidad de la onda de choque supera a la resistencia dinámica a la compresión " $\mathrm{RC}$ ", se produce una trituración de la roca circundante a las paredes del barreno por colapso de la estructura intercristalina."

Con estas indicaciones se calcularon los valores extremos para las resistencias dinámicas de la cuarcita y del gneis, obteniendo los valores siguientes:

Resistencias dinámicas máximas Cuarcita wollastonítica, MPa: $\mathrm{RC} / \mathrm{Rt}=2333 / 194,48=12$

Resistencias dinámicas mínimas Cuarcita wollastonítica, MPa: $\mathrm{RC} / \mathrm{Rt}=898 / 74,80=12$

Resistencias dinámicas máximas Gneis wollastonítico, MPa: $\mathrm{RC} / \mathrm{Rt}=2915,77 / 216,58=13,50$

Resistencias dinámicas mínimas Gneis wollastonítico, MPa: $\mathrm{RC} / \mathrm{Rt}=1121,45 / 83,30=13,50$

En conclusión, cuando se trata de analizar los efectos producidos por la voladura, conviene determinar las características dinámicas de las rocas, particularmente a tracción, puesto que el explosivo genera ese mismo tipo de esfuerzos, dinámicos mas no, estáticos. Para un uso racional de los explosivos, debe tenerse en mente que las condiciones impuestas por los explosivos son condiciones dinámicas y que por lo tanto demandan de cuantificar las condiciones dinámicas no de las rocas, sino de los macizos rocosos, para poder así hacer compatibles y reales los parámetros que deberían hacerse intervenir en el correcto diseño de una voladura y más de una voladura subterránea en donde las condiciones del macizo imponen características particulares.

De lo anterior se desprende la necesidad de conocer el macizo, a fin de poder evaluar su comportamiento real frente al uso de los explosivos para su fragmentación.

\subsection{Caracterización del macizo rocoso}

Los macizos rocosos (masas rocosas) están constituidos por la matriz rocosa o roca intacta y las discontinuidades, pero en un sentido más amplio puede incluirse igualmente el estado de esfuerzos in-situ; se presentan en la naturaleza y ambientes geológicos afectados por planos de discontinuidad o planos de debilidad que separan los bloques de matriz rocosa. Constituyen, al contrario de la matriz rocosa (que en la mayoría de los casos puede considerarse como un medio Continuo, homogéneo isotrópico y linealmente elástico -
(CHILE), un medio discontinuo, heterogéneo, anisotrópico e inelástico (DIANE) conformado en su conjunto tanto por bloques de matriz rocosa como por distintos tipos de discontinuidades que afectan al medio rocoso.

El conjunto de discontinuidades y bloques de matriz gobiernan el comportamiento mecánico global del macizo rocoso, a tal punto que para estudiar su comportamiento mecánico se deben analizar las propiedades de la matriz rocosa y de las discontinuidades.

Para caracterizar un macizo rocoso donde se pretende insertar una excavación, se requiere conocer los parámetros básicos de la roca y de las discontinuidades, así como la estructura del macizo que incluye aspectos como el número de familias de discontinuidades existentes, el espaciamiento medio de los planos de discontinuidad y las características geomecánicas básicas de las discontinuidades. Así mismo es necesario medir o estimar el estado tensional in-situ y en su caso las alteraciones producidas en el macizo por otras excavaciones.

Desde el punto de vista de su aplicación en ingeniería, las propiedades mécanicas de las rocas y de las discontinuidades geológicas, se pueden considerar suficientemente conocidas. Sin embargo los complejos entramados de rocas y discontinuidades que se han dado en llamar macizos rocosos, aunque se ha hecho un gran esfuerzo para investigarlos, todavia no se pueden considerar bien conocidos. Esta falta de conocimiento se debe en muchos casos a su inherente complejidad, a las dificultades de la observación de los macizos, siempre grandes, asi como a la heterogeneidad que les es propia [5].

El conocimiento de las propiedades de los macizos rocosos es posible hoy día gracias a las investigaciones adelantadas por investigadores de la talla del profesor Ever Hoek, (1997 y 2001) Ec. (5, 6 y 7), Ec. (12 y 13) y sus colaboradores, así como por los profesores Bieniawski Ec. (9 y 10), Ec. (14 y 15), Barton, Perri, (1999) Ec. (3 y 4), etc. Citados por Perri G. [6] quienes después de exhaustivas investigaciones tanto en campo como en laboratorio y oficina proponen relaciones entre las características de la roca intacta y las del macizo, teniendo en cuenta la influencia de las discontinuidades; a continuación, presentamos algunas de estas propiedades de los macizos rocosos.

$$
\begin{gathered}
\varphi_{m}=0.424 G S I-0.0016 G S I^{2}-6+9 \ln m_{i} \\
C_{m}=0.75 \sigma_{c i}\left(0.0058+0.0004 m_{i}\right) e^{G S i\left(0.0455-0.0073 \ln m_{i}\right)} \\
\sigma_{c m}=\left(0.0034 m_{i}^{0.8}\right) \sigma_{c i}\left[1.029+0.025 e^{\left(-0.1 m_{i}\right)}\right]^{G S I} \\
E_{m}=1000 \cdot \sqrt[2]{\left(\sigma_{c i} / 100\right)} \cdot 10^{(G S I-10) / 40} \\
K_{n}=E_{m}(1+v) / D \\
v_{m}=-0.002 \cdot G S I+v_{i}+0.2 \\
E_{M}=E_{R} \cdot e^{(0.0217-R M R \cdot 2.17)}
\end{gathered}
$$




$$
\begin{gathered}
E_{M}=E_{R} \cdot e^{(0.0564-R M R \cdot 5.64)} \\
I C=\sigma_{c m} / \gamma H
\end{gathered}
$$

Ec. (11) es válido para un túnel donde la profundidad es mayor que la altura del túnel $(\mathrm{H}>\mathrm{D})$

$\begin{array}{cl}\text { Donde: } & \\ \varphi_{m}: & \text { Ángulo de Fricción del macizo rocoso } \\ \mathrm{GSI}: & \text { Geological Strength Index } \\ m_{i}: & \text { Constante de las rocas según el GSI } \\ C_{m}: & \text { Cohesión del macizo rocoso } \\ \sigma_{c m}: & \text { Resistencia a compresión del macizo rocoso } \\ \sigma_{c i}: & \text { Resistencia a compresión de la roca intacta } \\ K_{n}: & \text { Rigidez de la discontinuidad } \\ v_{m}: & \text { Coeficiente de Poisson del macizo rocoso } \\ v_{i}: & \text { Coeficiente de Poisson de la roca intacta } \\ E_{M}: & \text { Módulo de Young del macizo rocoso } \\ E_{R}: & \text { Módulo de Young de la roca } \\ I C: & \text { Índice de competencia }\end{array}$

Resistencia a compresión del macizo rocoso:

$$
\sigma_{c m}=\sigma_{c i} \cdot S^{a}
$$

Resistencia a tracción biaxial del macizo rocoso:

Donde:

$$
\sigma_{t m}=-\frac{s \cdot \sigma_{c i}}{m_{b}}
$$

$\mathrm{S}, \mathrm{a}, m_{b}$ : parámetros de Hoek

$\sigma_{t m}: \quad$ resistencia a la tracción del macizo rocoso

Bieniawski [7] propone las siguientes expresiones para hallar el módulo de deformación del macizo rocoso:

$$
\begin{gathered}
E_{M}(G P a)=2 \cdot R M R-100 \\
E_{M}(G P a)=10^{\frac{R M R-10}{40}}
\end{gathered}
$$

Donde $E_{M}$ es el módulo de Young del macizo rocoso.

La primera de estas fórmulas es válida para $R M R>50$ y la segunda, para $R M R<50$. En macizos de peor calidad comienza a tener influencia el comportamiento de la roca intacta por lo que resulta más difícil realizar una propuesta suficientemente aproximada.

\subsection{Explosivos utilizados}

\begin{tabular}{|c|c|c|c|c|c|c|c|}
\hline \multicolumn{8}{|c|}{ Tipo de explosivo } \\
\hline \multicolumn{2}{|c|}{ ANFO } & \multicolumn{2}{|c|}{ Indugel Plus AP } & \multicolumn{2}{|c|}{ Emulind-E } & \multicolumn{2}{|c|}{ Emulind-B } \\
\hline $\begin{array}{c}\text { Densidad, } \\
\mathrm{g} / \mathrm{cm}^{3}\end{array}$ & $\begin{array}{c}\text { Velocidad } \\
\mathrm{m} / \mathrm{s}\end{array}$ & $\begin{array}{l}\text { Densidad, } \\
\mathrm{g} / \mathrm{cm}^{3}\end{array}$ & $\begin{array}{c}\text { Velocidad } \\
\mathrm{m} / \mathrm{s}\end{array}$ & $\begin{array}{c}\text { Densidad, } \\
\mathrm{g} / \mathrm{cm}^{3}\end{array}$ & $\begin{array}{c}\text { Velocidad } \\
\mathrm{m} / \mathrm{s}\end{array}$ & $\begin{array}{c}\text { Densidad, } \\
\mathrm{g} / \mathrm{cm}^{3}\end{array}$ & $\begin{array}{c}\text { Velocidad } \\
\mathrm{m} / \mathrm{s}\end{array}$ \\
\hline $0,85 \pm 0,05$ & $3000 \pm 300$ & $1,20 \pm 0,003$ & $4500 \pm 500$ & $1,16 \pm 0,06$ & $4600 \pm 600$ & $1,15 \pm 0,05$ & $5300 \pm 300$ \\
\hline
\end{tabular}

Los explosivos utilizados en nuestro medio son los producidos por INDUMIL, cuyas propiedades pueden consultarse en [8] del que se extrae la Tabla 1.

Tabla 1. Características de explosivos industriales, INDUMIL, 2018.

\subsection{Cálculo del Poder de Detonación}

La Presión de Detonación del Explosivo responde a la siguiente expresión:

$$
P_{D}=0.25 \cdot \rho_{e} \cdot V_{D}^{2} \cdot 10^{-3}
$$

Donde:

PD: presión de detonación del explosivo, $\mathrm{Mpa}$

$\rho_{\mathrm{e}}$ : densidad del explosivo, $\left(\mathrm{g} / \mathrm{cm}^{3}\right)$

$\mathrm{V}_{\mathrm{D}}$ : velocidad de detonación del explosivo, $\mathrm{m} / \mathrm{s}$

Según la teoría de la detonación en [9], la presión sobre las paredes del barreno para explosivos con densidad superior a $1 \mathrm{~g} / \mathrm{cm}^{3}$ se puede calcular como:

$$
P_{d}=\frac{\rho_{e} \cdot V_{D}^{2}}{8}
$$

Donde:

$\rho_{e}: \quad$ densidad del explosivo $\left(\mathrm{g} / \mathrm{cm}^{3}\right)$

$\mathrm{V}_{\mathrm{D}}$ : velocidad de detonación del explosivo $(\mathrm{km} / \mathrm{s})$

Para explosivos con densidad inferior a $1 \mathrm{~g} / \mathrm{cm}^{3}$ la presión sobre las paredes del barreno se calcula mediante:

$$
P_{d}=\frac{\rho_{e} \cdot V_{D}^{2}}{4.5}
$$

Estas ecuaciones suponen que el barreno está completamente lleno de explosivo; si el radio del barreno y el radio de la carga explosiva difieren o el barreno no está completamente lleno de explosivo, la presión sobre las paredes se calcula mediante la expresión:

$$
P_{h}=P_{d} \cdot\left(\frac{d_{e}}{d_{h}}\right)^{3}
$$

Donde:

$\mathrm{P}_{\mathrm{h}}$ : presión en las paredes del barreno $(\mathrm{GPa})$

$\mathrm{P}_{\mathrm{d}}$ : $\quad$ presión de detonación $(\mathrm{GPa})$

$\mathrm{d}_{\mathrm{e}}$ : diámetro de la carga explosiva

$\mathrm{d}_{\mathrm{h}}$ : diámetro del barreno

La ecuación (20) proporciona una herramienta muy útil para estimar la cantidad de explosivo necesaria para adquirir un valor de presión específico. A partir de esta ecuación se puede hallar también el diámetro de la carga explosiva.

$$
d_{e}=d_{h} \cdot \sqrt[3]{\frac{P_{h}}{P_{d}}}
$$

Por ejemplo, para lograr una presión de $0.5 \mathrm{GPa}$ en las paredes de los barrenos utilizando perforaciones de $0.102 \mathrm{~m}$ de diámetro y explosivos con una presión de detonación de 1.6 GPa, el diámetro de la carga explosiva debe ser $\sim 0.069$ $\mathrm{m}$ que corresponde a un diámetro estandarizado de $70 \mathrm{~mm}$ de explosivo patrón.

\section{Expresión propuesta}

Teniendo en cuenta el análisis anterior en donde hemos encontrado valores del burden con una dispersión bastante importante, para las condiciones del análisis de caso, y después de mucha investigación y análisis hemos concluido en la siguiente expresión (21), la cual estamos recomendando utilizar para voladuras subterráneas. 
En donde:

$$
B=\frac{K_{A} \times K_{S R F} \times K_{R M R b} \times D}{1000} \sqrt{\frac{P D}{R_{t d m}}}
$$

B: $\quad$ Burden, $m$

D: $\quad$ Diámetro, $\mathrm{mm}$

KSRF: Factor dependiente del SRF

$\mathrm{K}_{\mathrm{RMRb}}$ : Factor dependiente del RMRb

$\mathrm{K}_{\mathrm{A}}$ : $\quad$ Factor dependiente de la sección del túnel

PD: $\quad$ Poder de detonación del explosivo, MPa

*Rtdm: Resistencia a la tracción dinámica del macizo rocoso, $\mathrm{MPa}$

*Tomada como un porcentaje de la resistencia a la tracción dinámica de la roca intacta, pues hasta el momento no hemos encontrado una forma de determinar ese parámetro.

En la Tabla 2 se ilustran los valores del coeficiente para $\mathrm{K}_{\mathrm{A}}$, en la Tabla 3, los correspondientes a $\mathrm{K}_{\mathrm{SRF}}$, en la Tabla 4, los correspondientes a $\mathrm{K}_{\mathrm{RMRb}} \mathrm{y}$, finalmente, en la Tabla 5, los correspondientes a espaciamiento y retaque.

Tabla 2. Valores para $\mathrm{K}_{\mathrm{A}}$.

\begin{tabular}{|l|c|c|}
\hline \multicolumn{1}{|c|}{ Descripción } & $\mathbf{S T}\left(\mathbf{m}^{\mathbf{2}}\right)$ & $\mathbf{K}_{\mathbf{A}}$ \\
\hline Túneles de muy pequeña sección & $<20$ & 0,60 \\
\hline Túneles de pequeña sección & $20-40$ & 0,70 \\
\hline Túneles de mediana sección & $40-60$ & 0,80 \\
\hline Túneles de gran sección & $60-80$ & 0,90 \\
\hline Túneles de muy grande sección & $>80$ & 1,00 \\
\hline \multicolumn{2}{|c|}{ ST: Sección del túnel } \\
\hline
\end{tabular}

Tabla 3. Valores para $\mathrm{K}_{\mathrm{SRF}}$.

\begin{tabular}{|l|c|c|c|}
\hline \multicolumn{1}{|c|}{ Descripción } & SRF & P (m) & KRR \\
\hline Cerca de la superficie & ---- & $0-300$ & 1,00 \\
\hline Niveles de esfuerzos bajos & 2,5 & $300-600$ & 0,95 \\
\hline Niveles de esfuerzos medios & 1,0 & $600-900$ & 0,90 \\
\hline Niveles de esfuerzos altos & 0,5 & $>900$ & 0,85 \\
\hline \multicolumn{4}{|c}{ P: Profundidad }
\end{tabular}

Tabla 4. Valores para $\mathrm{K}_{\mathrm{RMRb}}$.

\begin{tabular}{|l|l|l|l|}
\hline \multicolumn{1}{|c|}{ Descripción } & \multicolumn{2}{|c|}{ RMRb } & K $_{\text {RMRb }}$ \\
\hline Macizo rocoso muy bueno & $80-100$ & I & 1,00 \\
\hline Macizo rocoso bueno & $60-80$ & II & 1,10 \\
\hline Macizo rocoso regular & $40-60$ & III & 1,20 \\
\hline Macizo rocoso malo & $20-40$ & IV & NA \\
\hline Macizo rocoso muy malo & $0-20$ & V & NA \\
\hline
\end{tabular}

Tabla 5. Valores para espaciamiento y retaque.

\begin{tabular}{|l|c|c|c|}
\hline RMRb & I & II & III \\
\hline K $_{\text {RMRb }}$ & 1,00 & 1,10 & 1,20 \\
\hline$\beta$ & 1,10 & 1,15 & 1,20 \\
\hline$\zeta$ & 0,40 & 0,60 & 0,80 \\
\hline LPRA (m) & $5,00-4,00$ & $4,00-3,00$ & $3,00-2,00$ \\
\hline
\end{tabular}

$\beta$ : Constante de espaciamiento); $\zeta$ : Constante de retaque; LPRA: Longitud promedia recomendada de avance

Una vez determinado el burden, los espaciamientos podrán hallarse de la siguiente forma dependiendo de la resistencia a la compresión de la roca y de la secuencia del retardo, pudiendo variar entre 1,2 (para rocas más resistentes y retardos más largos) y 1,0 (para rocas de menor resistencia y retardos más cortos).
Los retaques también serán función de la resistencia a la compresión de la roca y de la posición del barreno en la secuencia de encendido, así:

Para barrenos de producción, $\mathrm{T}=(0.4-0.8) \mathrm{B}$

Parra barrenos de piso, el retaque será, $\mathrm{T}=0.2 \mathrm{~B}$

Para barrenos de contorno, el retaque será, $\mathrm{T}=\mathrm{B}$

La Figura 1, ilustra los patrones de perforación y voladura, conforme los resultados de la expresión propuesta

\section{Características mecánicas de rocas colombianas}

\subsection{Generalidades}

La caracterización mecánica de las rocas se fundamenta en la determinación de sus propiedades de resistencia a la compresión simple y módulo de deformación, con lo cual se obtiene un tercer parámetro conocido como relación de módulos $E_{t} / \sigma_{c}$; con base en estos parámetros, resistencia a compresión uniaxial y relación de módulos, Deere y Miller, 1966 [10] propusieron su clasificación mecánica, Tablas 7 y 8.

Tabla 7. Clasificación de rocas según su resistencia a la compresión simple, fuente: [10].

\begin{tabular}{|c|c|c|}
\hline Categoría & RCS $(\mathrm{MPa})$ & Descripción \\
\hline A & $>2240$ & Resistencia extremadamente alta \\
\hline B & $1120-2240$ & Resistencia muy alta \\
\hline C & $560-1120$ & Resistencia alta \\
\hline D & $280-560$ & Resistencia media \\
\hline E & $140-280$ & Resistencia baja \\
\hline F & $<140$ & Resistencia muy baja \\
\hline
\end{tabular}

RCS: Resistencia a la Compresión Simple

Tabla 8. Clasificación de rocas según la relación de módulos, fuente: [10].

\begin{tabular}{|c|c|c|}
\hline Categoría & Relación de Módulos & Descripción \\
\hline $\mathbf{H}$ & $>500$ & Alta \\
\hline $\mathbf{L}$ & $200-500$ & Media \\
\hline $\mathbf{M}$ & $<200$ & Baja \\
\hline
\end{tabular}

En un trabajo de investigación realizado por el profesor Álvaro Correa [11] y del que se extrae la caracterización mecánica de rocas colombianas, se propone igualmente la clasificación de las rocas con base en el módulo de deformación, la cual se presenta en la Tabla 9.

Tabla 9. Clasificación de rocas, según módulo de deformación, fuente: [11].

\begin{tabular}{|c|c|c|}
\hline Categoría & Módulo de deformación $(\mathrm{GPa})$ & Descripción \\
\hline $\mathbf{1}$ & $>80$ & Rigidez extremadamente Alta \\
\hline $\mathbf{2}$ & $40-80$ & Rigidez muy Alta \\
\hline $\mathbf{3}$ & $20-40$ & Rigidez Alta \\
\hline $\mathbf{4}$ & $10-20$ & Rigidez media \\
\hline $\mathbf{5}$ & $5-10$ & Rigidez baja \\
\hline $\mathbf{6}$ & $<5$ & Rigidez muy baja \\
\hline
\end{tabular}

\subsection{Resumen, clasificación de rocas colombianas}

La recopilación de varios cientos de resultados de ensayos de laboratorio sobre rocas colombianas y su tratamiento permitió elaborar la Tabla 10. 
Tabla 10. Clasificación de las rocas colombianas según la metodología de Deere y Miller, fuente: [11].

\begin{tabular}{|c|c|c|}
\hline Tipo de roca & Clasificación & Muestras \\
\hline \multicolumn{3}{|c|}{ Rocas ígneas } \\
\hline Cuarzodiorita & $\mathrm{CH}, \mathrm{CM}$ & 33 \\
\hline Diabasa & $\mathrm{CM}$ & 50 \\
\hline \multicolumn{3}{|c|}{ Rocas Sedimentarias } \\
\hline Caliza & $\mathrm{DH}, \mathrm{DM}, \mathrm{CM}, \mathrm{CL}$ & 112 \\
\hline Arenisca & DH, CH para roca sana. AM, DM, CL para roca fisurada & 634 \\
\hline Limolita & $\mathrm{DM}, \mathrm{CM}, \mathrm{DL}, \mathrm{EM}$ & 111 \\
\hline Arcillolita & $\mathrm{DM}, \mathrm{CL}, \mathrm{EH}$ & 77 \\
\hline Argilita & $\mathrm{CM}, \mathrm{BM}, \mathrm{BL}$ & 94 \\
\hline Lutita & $\mathrm{DM}, \mathrm{EM}, \mathrm{EL}$ & 256 \\
\hline \multicolumn{3}{|c|}{ Rocas Metamórficas } \\
\hline Metalutita & $\mathrm{CH}, \mathrm{CM}$ & 17 \\
\hline Metasedimento & DM, DH & 15 \\
\hline Esquisto & DH, DM, CM & 113 \\
\hline Gneis & $\mathrm{CH}$ & 21 \\
\hline Cuarcita & $\mathrm{CM}, \mathrm{CL}, \mathrm{BL}$ & 50 \\
\hline
\end{tabular}

\subsection{Comparación clasificación mecánica de rocas}

Una comparación entre las propiedades mecánicas de la misma litología de rocas citadas por Deere y Miller y nuestras rocas, permite hacer la comparación que se ilustra en la Tabla 11.

Tabla 11. Comparación entre clasificaciones de rocas.

\begin{tabular}{|c|c|c|}
\hline Tipo de roca & Clasificación de Deere y Miller & Clasificación de rocas colombianas \\
\hline Limolita & CM, BM & DM, CM, DL, EM \\
\hline Arenisca & (B-D) M & (D-C) H \\
\hline Cuarcita & BM, AM & CM, CL, BL \\
\hline Gneis & BM, CM & CH \\
\hline Diabasa & AM & CM, BM \\
\hline
\end{tabular}

Según esta comparación, se aprecia que las rocas colombianas son más deformables y menos resistentes que las citadas por Deere y Miller [10] en sus investigaciones, lo que puede deberse a parámetros tales como la textura, estructura, composición mineralógica y porosidad, así como a la edad de la roca, las formaciones geológicas a las que pertenecen, la actividad sísmica y tectónica a las que han estado sometidas y la calidad de la roca (sana, fracturada, fisurada, etc.); por tanto, si se quiere llegar a confrontar datos más exactos, se deben tener en cuenta los parámetros anteriores.

De las cinco rocas en común, comparadas, se aprecia que en general los rangos de resistencia para las rocas investigadas por Deere y Miller oscilan entre A y B y los rangos de resistencia para las rocas colombianas se mantienen entre $C$ y E lo cual confirma las bajas resistencias de las rocas colombianas respecto a las citadas por Deere y Miller.

Para generar las regiones que se indican en las Figuras 2, 3 y 4 , en donde, se vaciaron los datos del diagrama original y se ubicó el centro sobre la masa de mayor concentración de las parejas de puntos $\left(\sigma_{\mathrm{c}} \mathrm{y} \mathrm{E}_{\mathrm{t}(50 \%)}\right)$, finalmente se tomó el $75 \%$ de las parejas de valores más cercanos en distancias reales al punto central seleccionado, para de esta manera poder trazar una envolvente que garantice que el $75 \%$ de las muestras se ubican dentro de la región, esta metodología se explica detalladamente en [11].

\section{Conclusiones y recomendaciones}

El burden es uno de los principales parámetros a determinar en una voladura tanto en superficie como subterráneamente.
Para su determinación, la bibliografía disponible cuenta con más de 20 expresiones, todas ellas propuestas con base en los seguimientos que sus autores han hecho en sus diferentes obras; es decir, son formulaciones empíricas. Estas expresiones, al ojo de un inexperto, lo primero que hacen es generar un cierto desconcierto al no saber cuál utilizar ni porqué.

Algunas de dichas expresiones son muy simples y solo hacen intervenir parámetros geométricos; otras, más elaboradas, recurren a caracterizar tanto la roca como el explosivo, y otras, las más sofisticadas se soportan en características tanto del explosivo como de la roca y del macizo rocoso. Como fruto de nuestras investigaciones en este campo, estamos proponiendo una expresión que hace intervenir las propiedades del explosivo, las características dinámicas de las rocas y las propiedades del macizo rocoso fundamentados en la determinación del RMRb, así como las condiciones del túnel respecto a su sección y localización subterránea. El éxito de una buena voladura depende de la supervisión de la perforación. La expresión propuesta tiene en cuenta las variables más importantes para el diseño de una voladura eficiente.

Para el caso colombiano se presentan algunas características físico-mecánicas de las rocas [8], con lo cual se espera que los resultados obtenidos con la expresión permitan ajustar las contantes y de esta manera realizar mejores diseños de perforación y voladuras. Poner a prueba esta propuesta con la finalidad de realizar mejoras y ajustes. Caracterizar más detenidamente el macizo rocoso con el fin de lograr unos mejores resultados en la voladura.

\section{Referencias}

[1] López E., López C., García P., Manual de perforación y voladura de rocas, Madrid, 2003.

[2] Peña M.F., "Calidad en el cálculo del Burden (B) basado en el modelo matemático de López Jimeno para contribuir en la gestión del riesgo en las voladuras, en cantera de roca caliza", Rev. del Instituto de Investigación FIGMMG-UNMSM, Vol. 22 (43) (2019), 53-58

[3] Ojeda R., "Diseño de mallas de perforación y voladura subterránea aplicando un modelo matematico de areas de influencia", Peru.

[4] López C., (1994). "Manual de perforación y voladura de rocas. Instituto Tecnológico GeoMinero de España”. Madrid.

[5] Ramírez P., Alejano L., (2004). "Mecánica de rocas. Fundamentos e ingeniería de taludes".

[6] Perri G., (2012). “Tópicos de ingeniería de túneles”. Caracas.

[7] Bieniawski Z.T., (1984) "Rock mechanics desing in mining and tunneling". A.A. Balkema.

[8] INDUMIL. (2018). "Manual para explosivos industriales"

[9] Slavko T., Veljko L., "Estimating extent and properties of blastdamaged zone around underground excavations", Rev. Esc. Minas 68 (4) (2015). 
[10] Deere D.U., Miller R.P., (1966). "Engineering classification and index properties for intact rock". Technical Report No. AFL-TR65-116 University of Illinois, Urbana, Illinois.
[11] Correa Arroyave Á., "Métodos recomendados por la ISRM para la caracterización de rocas en el laboratorio", Bogotá D.C: Sociedad Colombiana de Geotecnia, (2013).

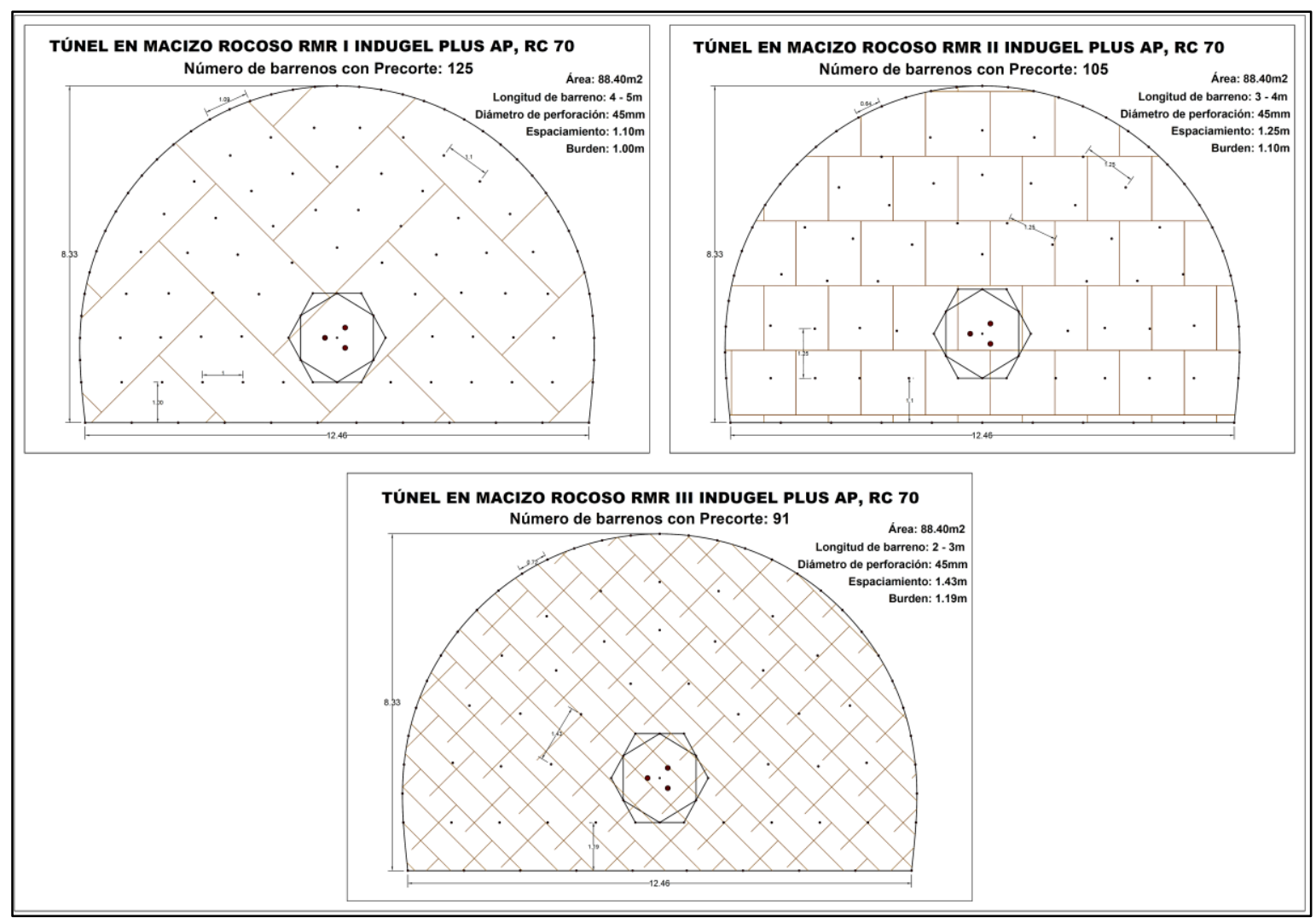

Figura 1. Esquemas de perforación y voladura, Fuentes Autores. 


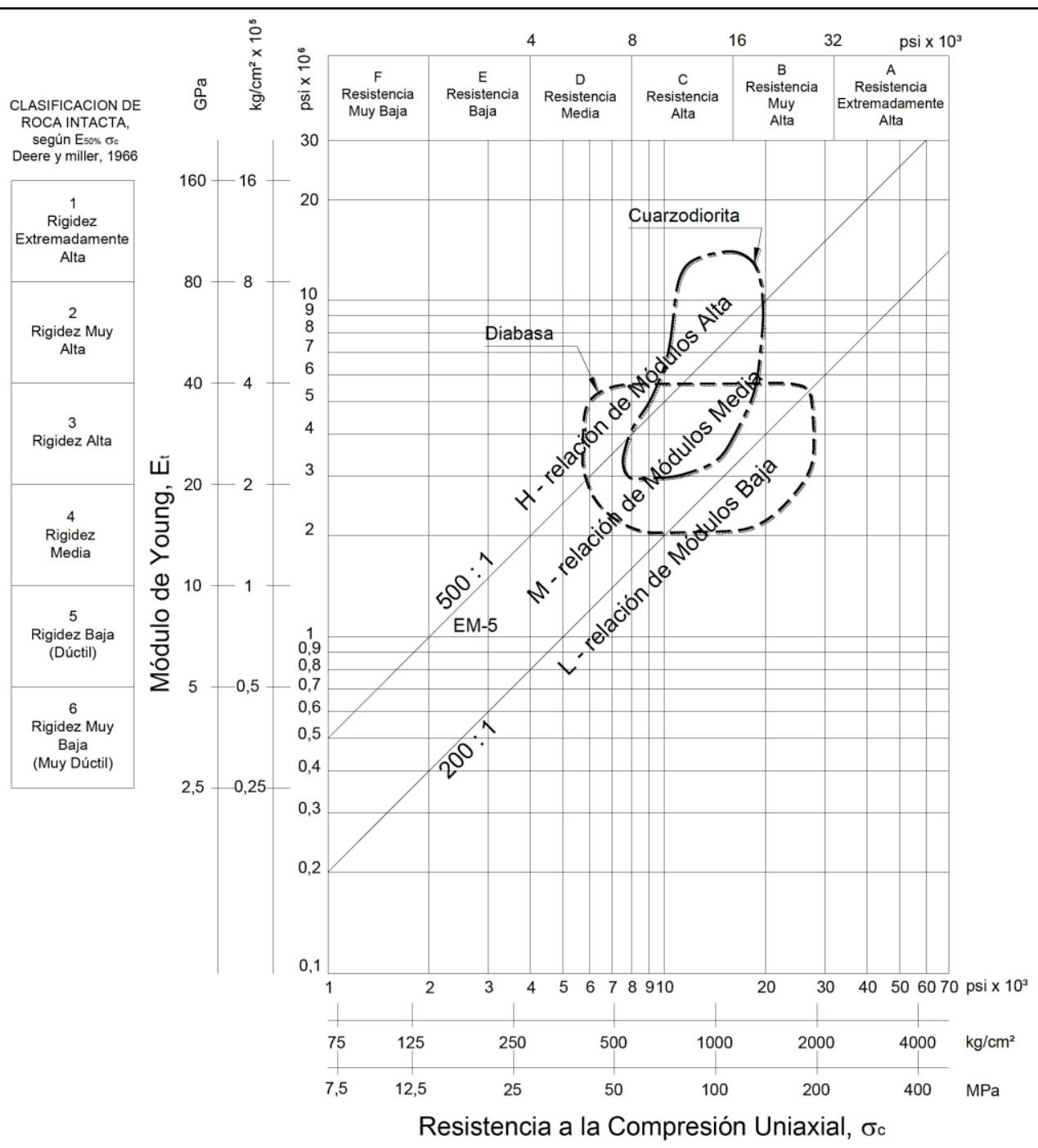

Figura 2. Rocas Ígneas, Fuente [11] 


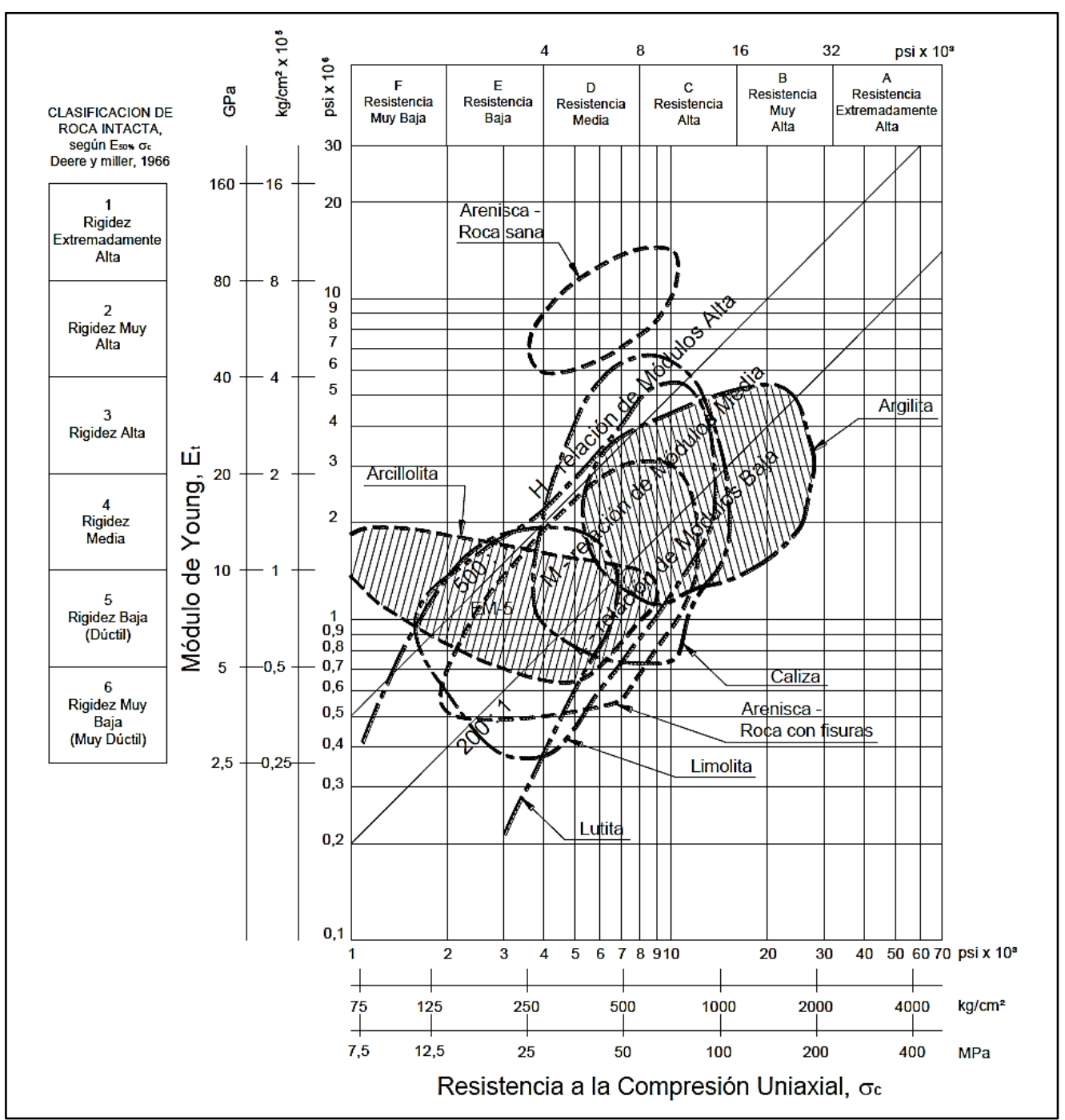

Figura 3. Rocas Sedimentarias, Fuente [11] 


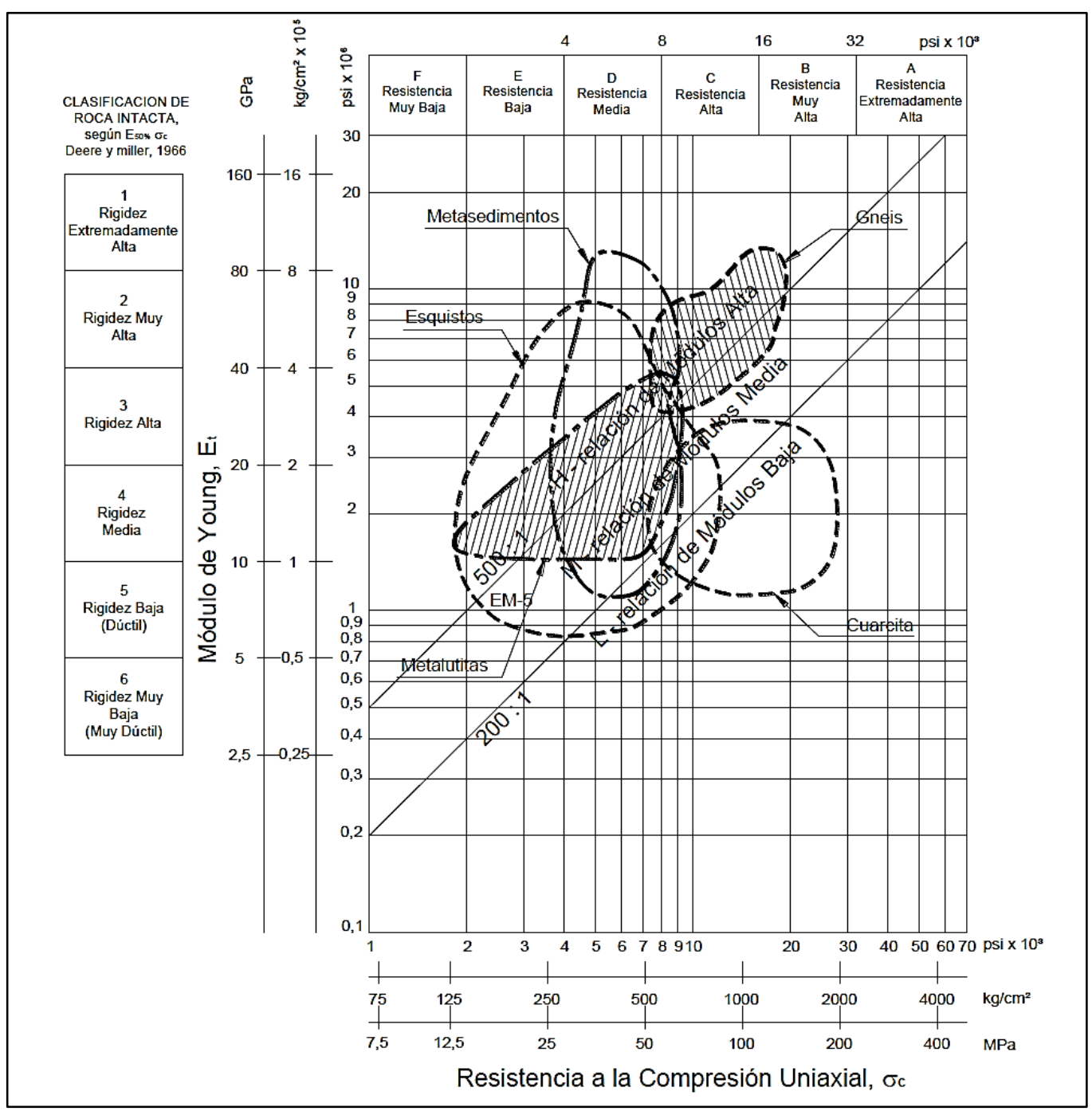

Figura 4. Rocas Metamórficas, Fuente [11]. 